\title{
Proportional modes versus pressure support ventilation: a systematic review and meta-analysis
}

Jun Kataoka ${ }^{{ }^{*} \mathbb{D}}$, Akira Kuriyama ${ }^{2}$, Yasuhiro Norisue ${ }^{1}$ and Shigeki Fujitani ${ }^{3}$

\begin{abstract}
Background: Proportional modes (proportional assist ventilation, PAV, and neurally adjusted ventilatory assist, NAVA) could improve patient-ventilator interaction and consequently may be efficient as a weaning mode. The purpose of this systematic review is to examine whether proportional modes improved patient-ventilator interaction and whether they had an impact on the weaning success and length of mechanical ventilation, in comparison with PSV.

Methods: We searched PubMed, EMBASE, and the Cochrane Central Register of Controlled Trials from inception through May 13, 2018. We included both parallel-group and crossover randomized studies that examined the efficacy of proportional modes in comparison with PSV in mechanically ventilated adults. The primary outcomes were (1) asynchrony index (Al), (2) weaning failure, and (3) duration of mechanical ventilation.
\end{abstract}

Results: We included 15 studies (four evaluated PAV, ten evaluated NAVA, and one evaluated both modes). Although the use of proportional modes was not associated with a reduction in $\mathrm{Al}$ (WMD $-1.43 ; 95 \% \mathrm{Cl}-3.11$ to 0.25 ;

$p=0.096 ;$ PAV — one study, and NAVA — seven studies), the use of proportional modes was associated with a reduction in patients with $\mathrm{Al}>10 \%$ (RR 0.15; 95\% Cl 0.04-0.58; $p=0.006$; PAV—-two studies, and NAVA—five studies), compared with PSV. There was a significant heterogeneity among studies for Al, especially with NAVA. Compared with PSV, use of proportional modes was associated with a reduction in weaning failure (RR $0.44 ; 95 \% \mathrm{Cl} 0.26-0.75 ; p=0.003$; PAV - three studies) and duration of mechanical ventilation (WMD -1.78 days; $95 \% \mathrm{Cl}-3.24$ to $-0.32 ; p=0.017$; PAV - three studies, and NAVA — two studies). Reduced duration of mechanical ventilation was found with PAV but not with NAVA.

Conclusion: The use of proportional modes was associated with a reduction in the incidence with $\mathrm{Al}>10 \%$, weaning failure and duration of mechanical ventilation, compared with PSV. However, reduced weaning failure and duration of mechanical ventilation were found with only PAV. Due to a significant heterogeneity among studies and an insufficient number of studies, further investigation seems warranted to better understand the impact of proportional modes.

Clinical trial registration PROSPERO registration number, CRD42017059791. Registered 20 March 2017

Keywords: Pressure support ventilation, Proportional assist ventilation, Neurally adjusted ventilator assist, Asynchrony index, Ventilator weaning, Systematic review, Meta-analysis

\footnotetext{
*Correspondence: rdcyc910@yahoo.co.jp

1 Department of Pulmonary and Critical Care Medicine, Tokyo Bay Urayasu

Ichikawa Medical Center, 3-4-32 Todaijima, Urayasu 2790001, Japan

Full list of author information is available at the end of the article
}

Springer Open

(c) The Author(s) 2018. This article is distributed under the terms of the Creative Commons Attribution 4.0 International License (http://creativecommons.org/licenses/by/4.0/), which permits unrestricted use, distribution, and reproduction in any medium, provided you give appropriate credit to the original author(s) and the source, provide a link to the Creative Commons license, and indicate if changes were made. 


\section{Introduction}

The separation of patient from the mechanical ventilator can take long time, and it is an important phase during the patient ventilator assistance [1]. Pressure support ventilation (PSV) is the most commonly used mode for the liberation process [2, 3], but presents several limitations. First, the optimal pressure support level for weaning varied among patients. Both over- and under-assistance may cause a diaphragm weakness [4]. Critical illness-associated diaphragm weakness is often associated with difficult weaning and prolonged duration of mechanical ventilation [5-7]. Second, PSV can often cause patient-ventilator asynchrony due to the mismatch between pressure support and the patient's inspiratory demand or effort level [8]. A recent study showed that the presence of asynchrony was associated with prolonged duration of mechanical ventilation and led to increased mortality [9].

Proportional assist ventilation (PAV) [10] and neurally adjusted ventilatory assist (NAVA) [11] are designed to improve patient-ventilator interaction [12]. Both modes are designed to adjust inspiratory pressure proportionally to the patient's inspiratory demand and are known as proportional modes [13]. PAV+ (Puritan Bennett 840/980 ventilator; Covidien, Boulder, Colorado, USA) automatically measures the elastance and resistance of the respiratory system during spontaneous breathing and delivers the adequate pressure needed to meet the flow and volume demand that are instantaneously measured on a breath-to-breath basis [14]. On the other hand, NAVA (Maquet Critical Care SA, S.Ina, Sweden) is controlled by the change of electrical activity of the diaphragm (EAdi) which is obtained by the placement of a nasogastric tube equipped with EMG electrodes [11]. Previous studies have demonstrated that both modes improve the patient-ventilator interaction [15]. Although proportional modes may be efficient as a weaning mode, they have not been examined on a large-scale randomized controlled trial compared to PSV as a weaning mode.

Therefore, we conducted a systematic review and meta-analysis to examine whether proportional modes improve patient-ventilator interaction and whether they have an impact on the weaning success and length of mechanical ventilation in mechanically ventilated patients, in comparison with PSV.

\section{Methods}

Our study protocol was registered at PROSPERO (CRD42017059791) on March 20, 2017. We complied with the PRISMA (Preferred Reporting Items for
Systematic Reviews and Meta-analyses) statement for reporting this systematic review [16].

\section{Eligibility criteria}

We included both parallel-group and crossover randomized studies that examined the efficacy of proportional modes (PAV and NAVA) in comparison with PSV in mechanically ventilated adults. We excluded studies that did not examined asynchrony index [17], pediatric and noninvasive ventilation studies, as well as parallelgroup studies that applied proportional modes only for spontaneous breathing trial.

\section{Database search}

We searched PubMed, EMBASE, and the Cochrane Central Register of Controlled Trials for eligible articles. Our search strategy was listed in Additional file 1: Table S1. Two authors (JK and AK) independently screened articles retrieved by the search and selected eligible articles. We also inspected the references of included studies for potentially relevant studies. In case of disagreement, the same authors consulted with a third author (YN). We placed no restrictions regarding publication status and languages. Our last search update was May 13, 2018.

\section{Data extraction and bias assessment}

Two authors (JK and AK) independently extracted the following data: (1) participants (age and proportion of females); (2) characteristics (country, type of ICUs, inclusion criteria of participants, parallel or crossover studies); (3) interventions (NAVA or PAV), and (4) outcomes of our interest listed below.

The same authors also independently assessed the risk of bias using Cochrane Risk of Bias tool [18]. Disagreement was resolved through discussion.

\section{Outcomes}

Our primary outcomes were (1) asynchrony index (AI), (2) weaning failure, and (3) duration of mechanical ventilation. The primary outcomes were analyzed for overall proportional modes of ventilation including NAVA and PAV together. AI was described in two different ways in the included studies. AI was either defined as a continuous outcome or as the number of patients with $\mathrm{AI}>10 \%$. We thus presented both definitions in our study. Weaning failure was generally defined as the need for switching to a controlled mode or reintubation after extubation. However, there is a significant heterogeneity in the definition of weaning failure among the studies. We thus included only studies with the definition of weaning failure of "extubation failure leading to reintubation." Our secondary outcomes included (1) weaning time from randomization, (2) switching again to a controlled mode, 
(3) length of stay in ICU, (4) length of stay in hospital, (5) ICU mortality, (6) in-hospital mortality, (7) patients who needed tracheostomy, (8) incidence of reintubation, and (9) those who required noninvasive ventilation after extubation.

\section{Statistical analysis}

We calculated risk ratios (RRs) for dichotomous outcomes and weighted mean difference (WMD) for continuous outcomes and presented the results with associated 95\% confidence intervals (CIs). Since many of the included studies reported continuous outcomes in medians and interquartile ranges, we converted these values to means and standard deviations using a method proposed by Wan et al. [19]. We pooled the data using a random-effects model [20]. There is no established method of pooling crossover studies. However, the pooled outcome of crossover studies is generally conservative than that of parallel studies. Although we acknowledge the unit-of-analysis error (double- or triplecounting studies and participants), we pooled all crossover studies as if they were parallel studies [18]. There is one three-way crossover study [15], and we evaluated the impact of this study by pooling only one comparison at a time in a sensitivity analysis. We assessed statistical heterogeneity with $I^{2}$ and $Q$ statistics [21]. We did not evaluate small study effect or publication bias according to the Cochrane methodology, because the number of studies included for each analysis was less than ten [18].

We conducted subgroup analysis by the type of intervention, namely NAVA and PAV, and examined the difference of outcomes between these subgroups with test of interaction. We also conducted sensitivity analysis, by excluding unclear or high risk of bias in sequence generation, allocation concealment, blinding of assessors, incomplete outcome reporting, and selective outcome reporting to assess the robustness of our primary outcome analyses.

We performed all analyses with Stata SE, version 15.0 (Stata, College Station, TX, USA). A threshold for statistical significance was set at $p<0.05$ (two-tailed).

\section{Results}

\section{Overview of included studies}

The search extracted 512 articles. After application of our inclusion and exclusion criteria, we considered seven parallel-group [22-28] and eight crossover [15, 29-35] studies that compared proportional modes with PSV in

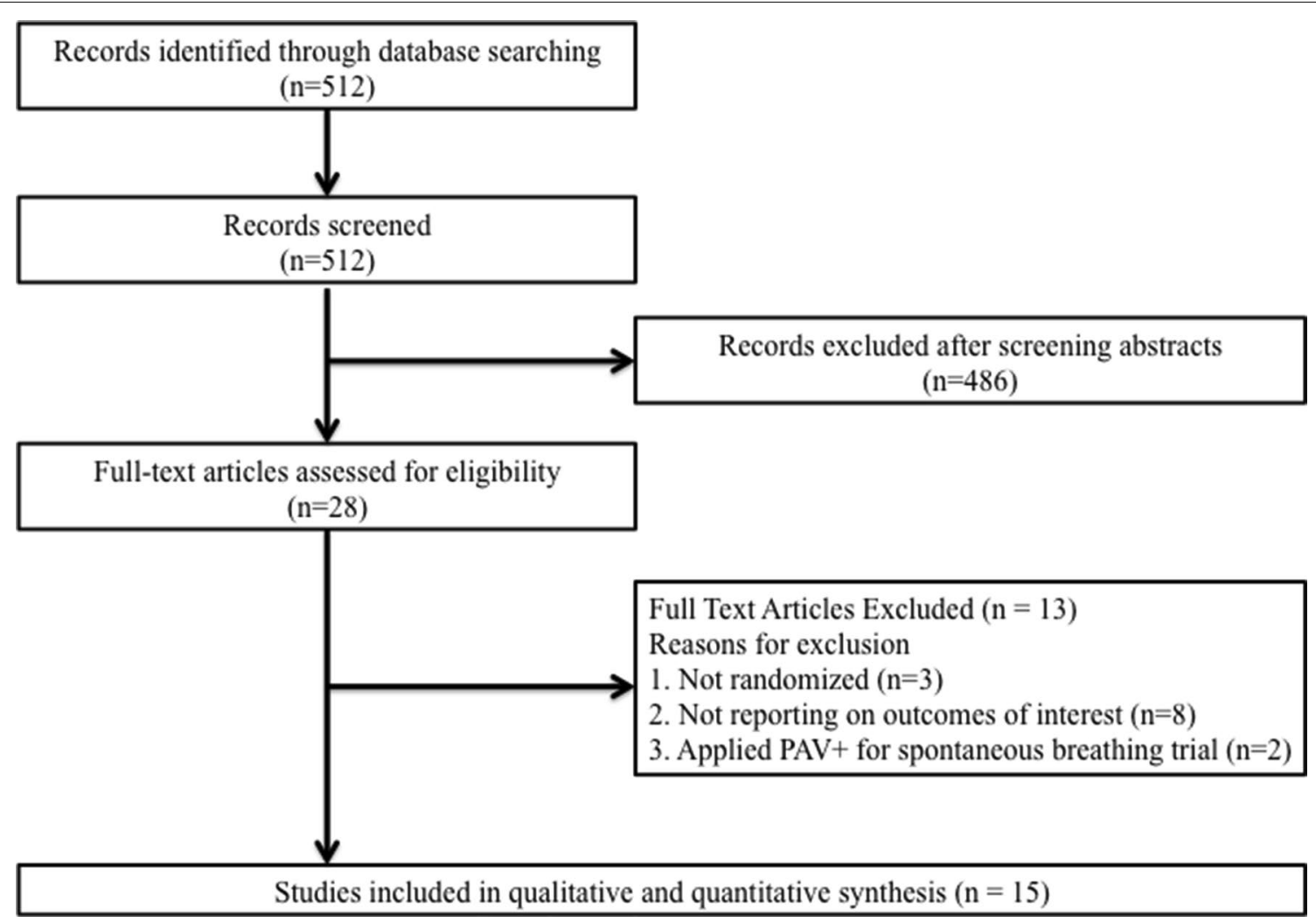

Fig. 1 Study selection 
mechanically ventilated adults (Fig. 1) (Additional file 2: Table S2). We contacted one study author to confirm that the participants were randomized, and therefore included this study in our analysis [33]. A total of 668 mechanical ventilated adult patients were included in the analysis (Table 1). The median or mean age of the participants ranged from 55.4 to 77.9 years, and $34.1 \%$ were women. Four studies evaluated PAV $(n=367)$ [22-24, 28], ten studies evaluated NAVA $(n=285)$ [25-27, 29-35], and one crossover study evaluated both modes $(n=16)$ [15]. We included the whole literature on proportional assist ventilation. However, we could not find studies that met our inclusion criteria other than examined PAV + . One parallel study recruited multicenter ICUs [25], six studies recruited medical ICUs [23, 27, 31-33, 35], one crossover study recruited surgical ICU [34], and seven studies recruited mixed (medical and surgical) ICUs [15, 22, 24, 26, 28-30]. All studies were published in full texts and in English.

\section{Risk of bias}

Sequence generation and allocation concealment were adequately conducted in ten $(67 \%)[15,22-25,28,29$, $33-35]$ and five studies (33\%) [23, 25, 28, 34, 35], respectively (Table 2). Blinding of participants and investigators was impossible due to the nature of study design, but blinding of outcome assessors was deemed appropriate in two studies (13\%) $[25,35]$. Nine studies $(60 \%)$ were free of the risk of incomplete outcome reporting [22, 24, 27$29,31,33-35]$. Since many of the included studies were crossover studies that failed to report that baseline data between groups, only four studies (27\%) were considered at low risk of other bias [22-25].

\section{Primary outcomes}

Asynchrony index

Although the use of proportional modes was not associated with a reduction in AI (WMD - 1.43; 95\% CI - 3.11 to $0.25 ; p=0.096 ; d f=7 ; I^{2}=82.4 \%$ ) (Fig. $2 \mathrm{a}$ ), the use of proportional modes was associated with a reduction in patients with $\mathrm{AI}>10 \%$ ( $\mathrm{RR} 0.15$; 95\% CI 0.04-0.58; $p=0.006 ; d f=6 ; I^{2}=61.2 \%$ ) (Fig. 2b), compared with PSV. In the subgroup analysis, the use of NAVA was associated with a reduction in AI. There was a significant heterogeneity among studies for AI, especially with NAVA.

\section{Weaning failure}

Three studies were included in the analysis, and all of them compared PAV and PSV [22, 23, 28]. The definition of weaning failure in each study is shown in Table 1 . The use of proportional modes was associated with reduction in weaning failure (RR 0.44; 95\% CI 0.26-0.75; $p=0.003$; $d f=2 ; I^{2}=0.0 \%$ ) (Fig. 3), compared with PSV.

\section{Duration of mechanical ventilation}

Five studies reported duration of mechanical ventilation (three and two studies evaluated PAV and NAVA, respectively) [22-25, 27]. The use of proportional modes was associated with a shorter duration of mechanical ventilation (WMD -1.78 days; $95 \% \mathrm{CI}-3.24$ to -0.32 ; $\left.p=0.017 ; d f=4 ; I^{2}=32.5 \%\right)$ in comparison with PSV (Fig. 4). In the subgroup analysis, the use of PAV was associated with a reduction in duration of mechanical ventilation in comparison with PSV, while there was no such association for NAVA.

\section{Secondary outcomes}

Compared with PSV, proportional modes did not exhibit any association with reduction in weaning time from randomization, switching again to a controlled mode, length of ICU stay, length of hospital stay, hospital mortality or tracheostomy, compared with use of PSV (Table 3). However, the use of proportional modes was significantly associated with reduced incidence of reintubation (RR $0.39 ; 95 \%$ CI $\left.0.17-0.90 ; p=0.027 ; d f=2 ; I^{2}=0.0 \%\right)$ and the use of noninvasive ventilation after extubation (RR $0.64 ; 95 \%$ CI $\left.0.47-0.89 ; p=0.007 ; d f=1 ; I^{2}=0.0 \%\right)$ in comparison with PSV (Table 3).

\section{Subgroup analysis}

We conducted subgroup analyses on the primary outcomes (Table 4). There was no significant difference between NAVA and PAV groups in any of the primary outcomes.

\section{Sensitivity analysis}

We conducted sensitivity analyses on the primary outcomes (Additional file 3: Table S3). Although some sensitivity analysis was impossible for secondary outcomes due to the lack of low-risk studies, other available sensitivity analyses were mostly consistent with the primary outcome analysis. Pooling only one comparison from a three-way crossover study for AI yielded finding similar to the primary analysis.

\section{Discussion}

The present study provides the following findings: (1) proportional modes reduced the incidence with $\mathrm{AI}>10 \%$, and (2) the use of proportional modes, especially PAV, was associated with a reduction in weaning failure and duration of mechanical ventilation, compared with PSV. Sensitivity analyses corroborated the robustness of our findings, and despite the small sample size within each of the included studies, our systematic review and metaanalysis suggests that proportional modes may have some merits for patients undergoing liberation from mechanical ventilation, compared with PSV. 


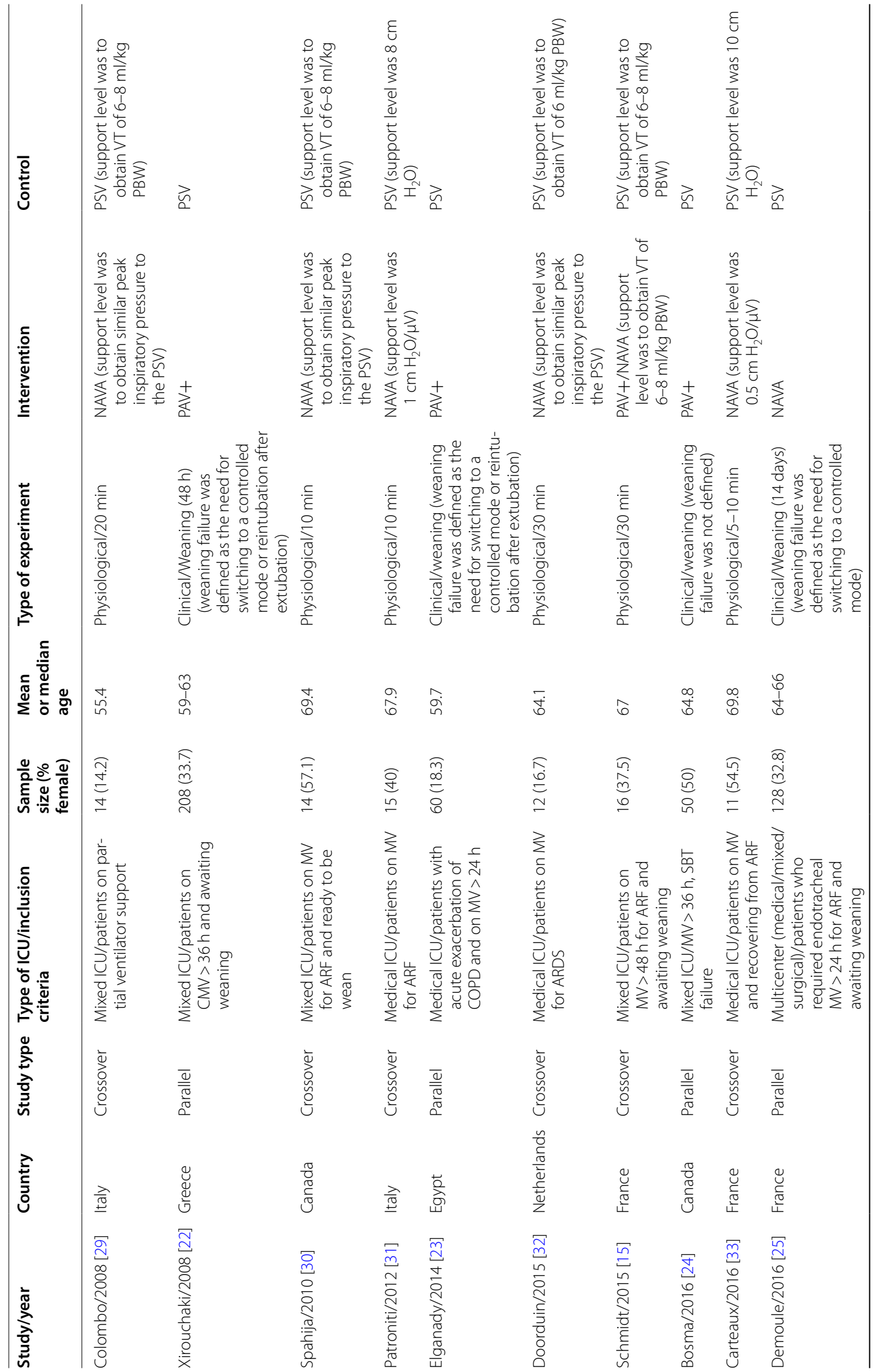




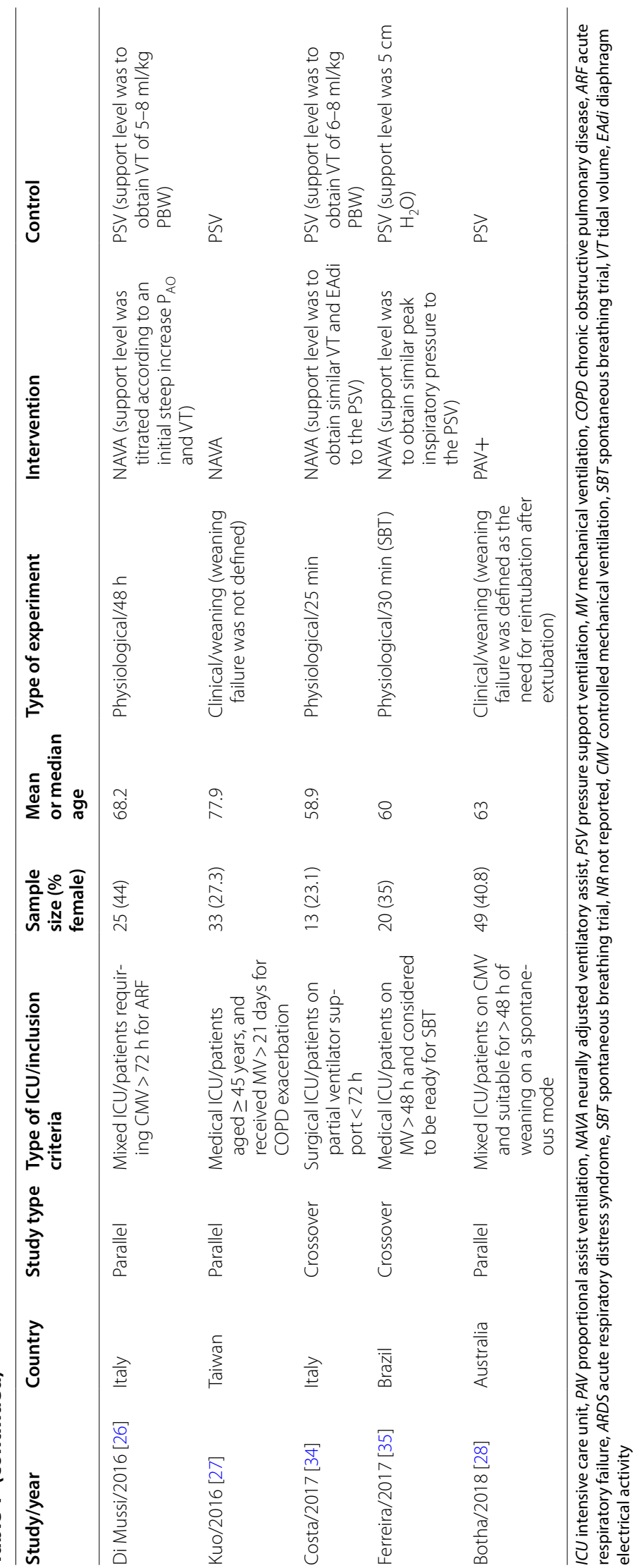


Table 2 Risk of bias in included studies

\begin{tabular}{|c|c|c|c|c|c|c|c|}
\hline Study/year & $\begin{array}{l}\text { Sequence } \\
\text { generation }\end{array}$ & $\begin{array}{l}\text { Allocation } \\
\text { concealment }\end{array}$ & $\begin{array}{l}\text { Blinding } \\
\text { of participants } \\
\text { and personnel }\end{array}$ & $\begin{array}{l}\text { Blinding } \\
\text { of outcome } \\
\text { assessors }\end{array}$ & $\begin{array}{l}\text { Incomplete } \\
\text { outcome data }\end{array}$ & $\begin{array}{l}\text { Selective } \\
\text { outcome } \\
\text { reporting }\end{array}$ & $\begin{array}{l}\text { Other } \\
\text { source } \\
\text { of bias }\end{array}$ \\
\hline Colombo/2008 & Low & Unclear & High & Unclear & Low & Unclear & Unclear \\
\hline Xirouchaki/2008 & Low & Unclear & High & Unclear & Low & Low & Low \\
\hline Spahija/2010 & Unclear & Unclear & High & Unclear & Unclear & Unclear & Unclear \\
\hline Patroniti/2012 & Unclear & Unclear & High & Unclear & Low & Unclear & Unclear \\
\hline Elganady/2014 & Low & Low & High & Unclear & Unclear & Low & Low \\
\hline Doorduin/2015 & Unclear & Unclear & High & Unclear & Unclear & Low & Unclear \\
\hline Schmidt/2015 & Low & Unclear & High & Unclear & Unclear & Low & Unclear \\
\hline Bosma/2016 & Low & Unclear & High & Unclear & Low & Unclear & Low \\
\hline Carteaux/2016 & Low & Unclear & High & High & Low & Low & Unclear \\
\hline Demoule/2016 & Low & Low & High & Low & High & Low & Low \\
\hline Di mussi/2016 & Unclear & Unclear & High & Unclear & High & High & Unclear \\
\hline Kuo/2016 & Unclear & Unclear & High & Unclear & Low & Low & Unclear \\
\hline Costa/2017 & Low & Low & High & Unclear & Low & Low & Unclear \\
\hline Ferreira/2017 & Low & Low & High & Low & Low & Low & Unclear \\
\hline Botha/2018 & Low & Low & High & High & Low & Low & High \\
\hline
\end{tabular}

Our study showed that proportional modes significantly reduced the incidence with $\mathrm{AI}>10 \%$, compared to PSV. The significant reduction of the incidence with $\mathrm{AI}>10 \%$ is mainly attributed to the existence of neuromuscular coupling in proportional modes, allowing the patient to have control over the airway pressure provided by the ventilator according to the patient's inspiratory demand. Over-assistance with PSV generated an important prevalence of ineffective effort. For instance, Schmidt et al. showed that PAV and NAVA improved patient-ventilator interaction by preventing over-distention and ineffective effort in their preliminary crossover study [15]. Although several patient-ventilator asynchronies classifications exist [36], we were unable to identify which classifications of asynchrony actually improved with each mode because AI was solely utilized as an outcome. PAV and NAVA may have different profiles in preventing asynchrony such as double triggering which was observed more frequently in NAVA than in both PAV and PSV [15]. On the other hand, inspiratory trigger delay was observed less frequently in NAVA than in both PAV and PSV [15].

Remarkably, our study showed that the use of proportional modes, especially PAV, was significantly associated with reduced weaning failure and duration of mechanical ventilation. The possible explanations for the finding are as follows. First, the patients with PSV may have been given more frequent or higher doses of analgesics or sedatives due to higher incidence of asynchrony. Previous studies showed that proportional modes could not only improve sleep quality [37] but also decrease the dose of sedative medication [24] because of better patient-ventilator interaction. Maintaining light levels of sedation is shown to be associated with shorter duration of mechanical ventilation [38, 39]. Second, asynchronies such as ineffective effort and double triggering may have unfavorable effects on patients' respiratory systems, which lead to longer duration of mechanical ventilation on PSV. Third, proportional modes may reduce risk of over- and under-assistance. PAV+ monitors the work of breathing and inspiratory respiratory effort of the patients [40], while NAVA monitors the electrical activity of diaphragm and thus may minimize diaphragmatic atrophy due to inactivity. In fact, over-assistance is a common scenario with PSV and leads to diaphragm atrophy, explaining the increased duration of mechanical ventilation [41, 42]. However, these assumptions have been somewhat controversial. Two multicenter RCTs are presently under way (NCT02447692, NCT01730794), and their results will presumably become available in the near future. Our meta-analysis supports, while awaiting the results of the $\mathrm{RCTs}$, that it is reasonable to use proportional modes in the liberation process for mechanically ventilated adults.

Finally, we determined that proportional modes were associated with less frequent application of post-extubation noninvasive ventilation and reintubation. Each study did not include predefined criteria for post-extubation noninvasive ventilation and reintubation. Due to a significant heterogeneity among studies and an insufficient number of studies, further investigation seems warranted to better understand the impact of proportional modes on secondary outcomes. 


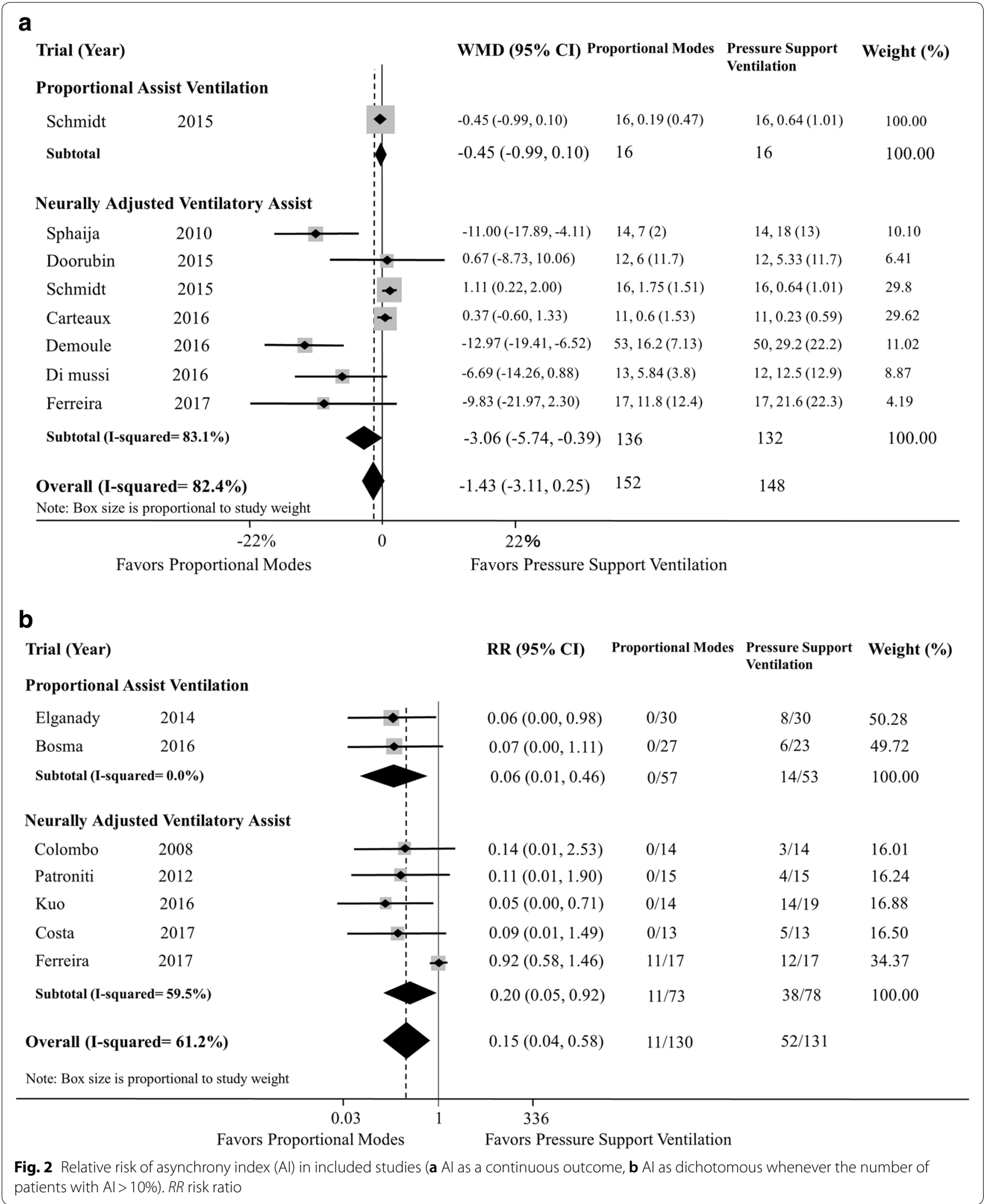




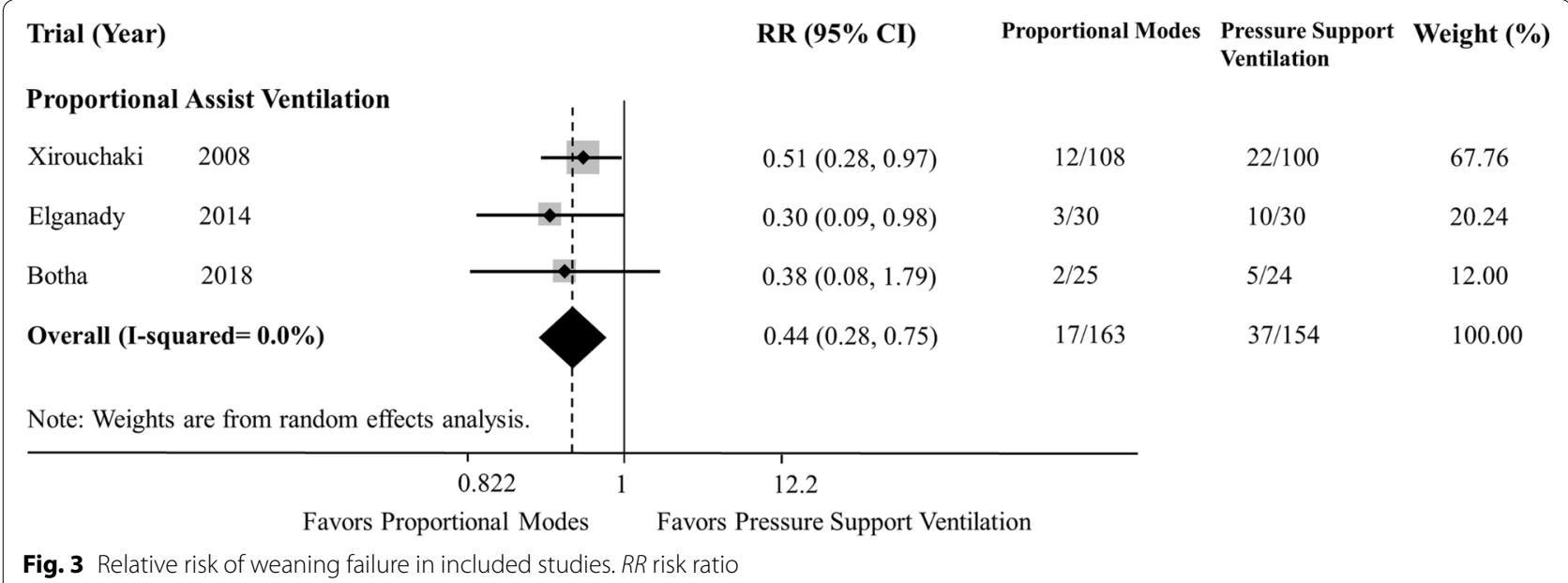

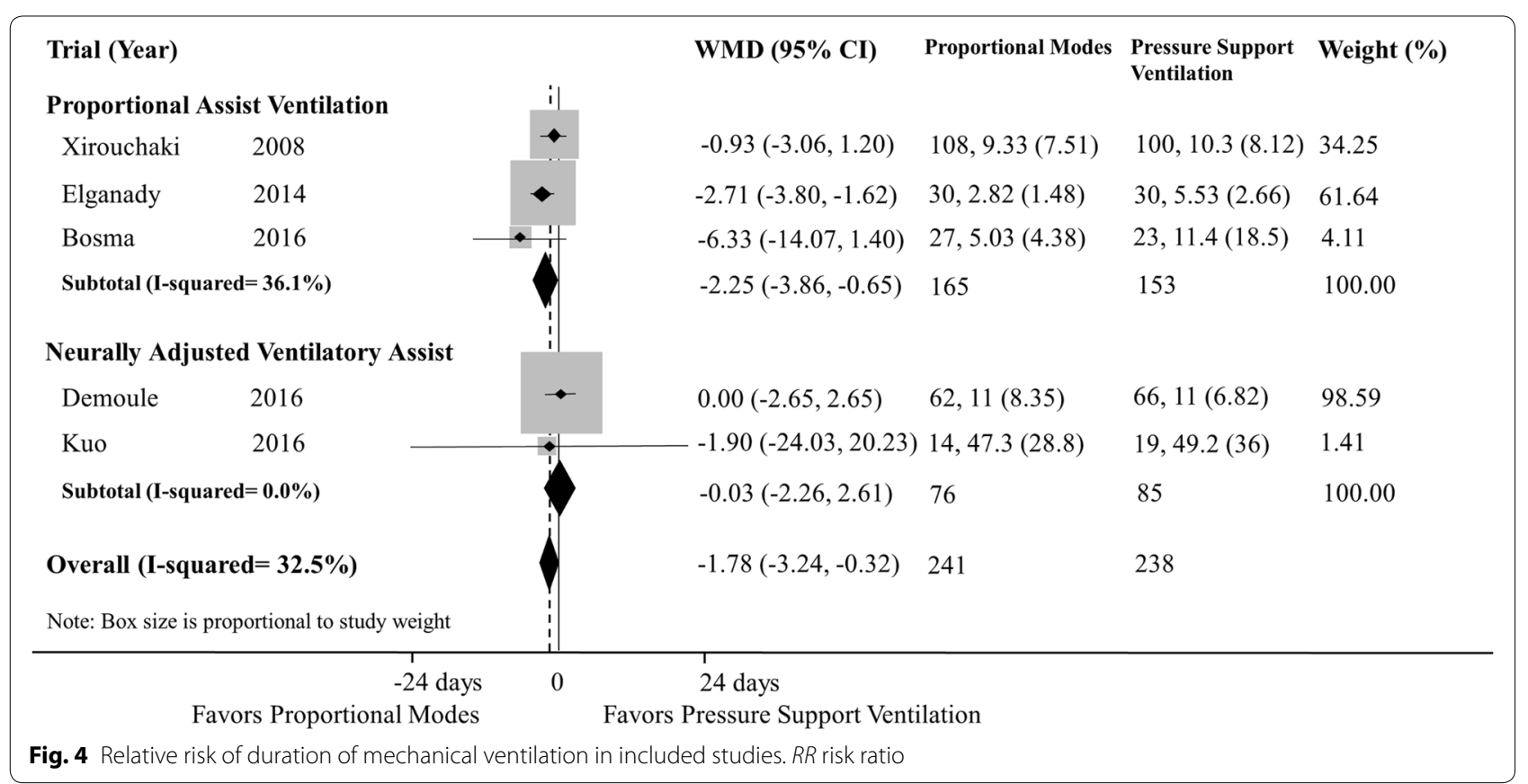

Our study has several strengths. First, to the best of our knowledge, this represents the first systematic review and meta-analysis that examines the efficacy of proportional modes as weaning modes. A comprehensive search was conducted, and fifteen studies were included. This study could reveal the efficacy of proportional modes as weaning modes, compared to PSV. Second, this systematic review examined a broad array of outcomes and hence could propose future pertinent proportional modes investigations to be conducted not only on weaning but also post-extubation outcomes, namely the utilization of noninvasive ventilation and reintubation. Third, subgroup analysis (PAV or NAVA) could be conducted due to the large number of studies.

Our study also has some limitations. First, both PAV and NAVA were concurrently analyzed since both have similar objectives. However, PAV and NAVA have some differences and thus are not completely the same. For instance, PAV delivers the support proportionally to lung mechanics, while NAVA cannot [43]. Therefore, there may be some differences in outcomes between PAV and NAVA in patients with abnormal respiratory system mechanics. Although a comparison of PAV and NAVA is thus clinically relevant, we did not conduct a network 
Table 3 Results of secondary outcomes

\begin{tabular}{|c|c|c|c|c|c|c|}
\hline Outcomes & No. of trials (PAV/NAVA) & $\begin{array}{l}\text { Total } \\
\text { sample size }\end{array}$ & $\begin{array}{l}\text { Summary estimates ( } 95 \% \\
\text { confidence intervals) }\end{array}$ & $Q$ & $d f$ & $I^{2}(\%)$ \\
\hline Weaning time from randomization (day) & $\begin{array}{l}\text { PAV } 2[24,28] \\
\text { NAVA } 1[25]\end{array}$ & 220 & WMD $-1.21(-4.32,1.91)$ & 3.66 & 2 & 45.3 \\
\hline Switching again to a controlled mode & $\begin{array}{l}\text { PAV } 1[24] \\
\text { NAVA } 1 \text { [25] }\end{array}$ & 168 & RR $1.00(0.77,1.31)$ & 0.30 & 1 & 0.0 \\
\hline Length of ICU (day) & $\begin{array}{l}\text { PAV } 4[22-24,28] \\
\text { NAVA } 2[25,27]\end{array}$ & 528 & WMD $-1.41(-3.90,1.09)$ & 12.43 & 5 & 59.8 \\
\hline Length of hospital stay (day) & $\begin{array}{l}\text { PAV } 3[23,24,28] \\
\text { NAVA } 2[25,27]\end{array}$ & 320 & WMD $-0.26(-3.90,3.37)$ & 7.73 & 4 & 48.2 \\
\hline Tracheostomy & PAV $2[24,28]$ & 98 & RR $0.65(0.31,1.37)$ & 0.09 & 1 & 0.0 \\
\hline Reintubation & PAV $3[23,24,28]$ & 158 & RR $0.39(0.17,0.90)$ & 0.39 & 2 & 0.0 \\
\hline Use of NIV after extubation & $\begin{array}{l}\text { PAV } 1 \text { [24] } \\
\text { NAVA } 1[25]\end{array}$ & 178 & RR $0.64(0.47,0.89)$ & 0.13 & 1 & 0.0 \\
\hline
\end{tabular}

$R R$ risk ratio, $W M D$ weighted mean difference, $I C U$ intensive care unit, $P A V$ proportional assisted ventilation, NAVA neurally adjusted ventilatory assist

Table 4 Subgroup analyses of primary outcomes

\begin{tabular}{|c|c|c|c|c|c|c|c|}
\hline \multirow[t]{2}{*}{ Outcomes } & \multirow{2}{*}{$\begin{array}{l}\text { No. of trials } \\
\text { (PAV/NAVA) }\end{array}$} & \multirow{2}{*}{$\begin{array}{l}\text { Total } \\
\text { sample } \\
\text { size }\end{array}$} & \multirow{2}{*}{$\begin{array}{l}\text { Summary estimates (95\% } \\
\text { confidence intervals) }\end{array}$} & \multicolumn{3}{|c|}{ Heterogeneity } & \multirow{2}{*}{$\begin{array}{l}p \text { value (test } \\
\text { for subgroup } \\
\text { difference) }\end{array}$} \\
\hline & & & & $Q$ & $d f$ & $I^{2}(\%)$ & \\
\hline \multirow[t]{2}{*}{ Al as continuous outcome } & $\mathrm{PAV}, 1$ & 32 & WMD $-0.45(-1.00,0.10)$ & 0.00 & 0 & - & \multirow[t]{2}{*}{0.06} \\
\hline & NAVA, 7 & 268 & WMD $-3.06(-5.74,-0.39)$ & 35.53 & 6 & 83.1 & \\
\hline \multirow[t]{2}{*}{$\mathrm{Al}>10 \%$} & PAV, 2 & 110 & RR $0.06(0.01,0.46)$ & 0.00 & 1 & 0.0 & \multirow[t]{2}{*}{0.57} \\
\hline & NAVA, 5 & 151 & $\operatorname{RR} 0.20(0.05,0.92)$ & 15.47 & 4 & 59.5 & \\
\hline Weaning failure & PAV, 3 & 317 & $\operatorname{RR} 0.44(0.26,0.75)$ & 0.60 & 2 & 0.0 & NA \\
\hline \multirow[t]{2}{*}{ Duration of mechanical ventilation } & PAV, 3 & 318 & WMD $-2.25(-3.86,-0.65)$ & 3.13 & 2 & 36.5 & \multirow[t]{2}{*}{0.16} \\
\hline & NAVA, 2 & 161 & WMD $-0.03(-2.66,2.61)$ & 0.03 & 1 & 0.0 & \\
\hline
\end{tabular}

$A$ l asynchrony index, $R R$ risk ratio, $W M D$ weighed mean difference

meta-analysis to compare PAV and NAVA for the following reasons: (1) the number of studies included in each analysis was limited; (2) a network meta-analysis is not recommended when there were presumably clinical heterogeneity across studies; and (3) the subgroup analysis already found no statistical difference between the two subgroups. Second, each included study in this systematic review utilized the differential weaning protocol which may possibly affect the duration of mechanical ventilation. Third, there was a significant heterogeneity among studies for AI, especially with NAVA, but an insufficient number of studies precluded the analysis to investigate the source of this heterogeneity. Fourth, the risk of bias in many of the included studies was deemed high. However, although it was impossible to perform some sensitivity analyses due to the lack of studies at low risk of certain bias, most available sensitivity analyses produced findings similar to the primary analysis, which further made the analysis more rigorous. Fifth, many of the included studies were crossover studies. They have a theoretical risk that the efficacy of the first intervention may be overestimated or underestimated in comparison with that of the second one [44], because the patients were supposed to gradually improve during the weaning period considered in the included studies.

\section{Conclusion}

The use of proportional modes was associated with a reduction in the incidence with $\mathrm{AI}>10 \%$, weaning failure and duration of mechanical ventilation, compared with PSV. However, reduced weaning failure and duration of mechanical ventilation were found with only PAV. Due to a significant heterogeneity among studies and an insufficient number of studies, further investigation seems warranted to better understand the impact of proportional modes.

\section{Additional files}

Additional file 1: Table S1. Search strategy. 
Additional file 2: Table S2. Full text articles excluded.

Additional file 3: Table S3. Sensitivity analyses of primary outcomes.

\section{Abbreviations}

PSV: pressure support ventilation; PAV: proportional assist ventilation; NAVA: neurally adjusted ventilatory assist; RCT: randomized controlled trial; Al: asynchrony index; EAdi: electrical activity of the diaphragm; RR: risk ratio; $\mathrm{Cl}$ : confidence interval; WMD: weighted mean difference.

\section{Authors' contributions}

JK and AK conceived the study and developed the study design. JK and AK performed the study search, extracted data, carried out statistical analysis, drafted the manuscript, and revised the manuscript. YN and SF improved the idea and design and revised the manuscript. All authors read and approved the final manuscript.

\section{Author details}

1 Department of Pulmonary and Critical Care Medicine, Tokyo Bay Urayasu Ichikawa Medical Center, 3-4-32 Todaijima, Urayasu 2790001, Japan.

2 Emergency and Critical Care Center, Kurashiki Central Hospital, 1-1-1 Miwa, Kurashiki, Okayama 7108602, Japan. ${ }^{3}$ Department of Emergency Medicine and Critical Care Medicine, St. Marianna University, 2-16-1 Sugao, Miyamae-ku, Kawasaki 2168511, Japan.

\section{Acknowledgements}

We thank Professor Dimitris Georgopoulos for his helpful advice on the discussion. We thank Dr. Brandon Lohman for his medical English proofreading.

\section{Competing interests}

The authors declare that they have no competing interests.

\section{Availability of data and materials}

The datasets used and/or analyzed during the current study are available from the corresponding author on reasonable request.

\section{Consent for publication}

Not applicable.

\section{Ethics approval and consent to participate}

Not applicable.

\section{Funding}

The authors received no specific funding for this work.

\section{Publisher's Note}

Springer Nature remains neutral with regard to jurisdictional claims in published maps and institutional affiliations.

Received: 21 August 2018 Accepted: 4 December 2018 Published online: 10 December 2018

\section{References}

1. Boles JM, Bion J, Connors A, Herridge M, Marsh B, Melot C, et al. Weaning from mechanical ventilation. Eur Respir J. 2007;29(5):1033-56.

2. Esteban A, Alia I, Ibanez J, Benito S, Tobin MJ. Modes of mechanical ventilation and weaning. A national survey of Spanish hospitals. The Spanish Lung Failure Collaborative Group. Chest. 1994;106(4):1188-93.

3. Esteban A, Anzueto A, Frutos F, Alia I, Brochard L, Stewart TE, et al. Characteristics and outcomes in adult patients receiving mechanical ventilation: a 28-day international study. JAMA. 2002;287(3):345-55.

4. Dres M, Goligher EC, Heunks LMA, Brochard LJ. Critical illness-associated diaphragm weakness. Intensive Care Med. 2017:43(10):1441-52.
5. Kim WY, Suh HJ, Hong SB, Koh Y, Lim CM. Diaphragm dysfunction assessed by ultrasonography: influence on weaning from mechanical ventilation. Crit Care Med. 2011;39(12):2627-30.

6. Jung B, Moury PH, Mahul M, de Jong A, Galia F, Prades A, et al. Diaphragmatic dysfunction in patients with ICU-acquired weakness and its impact on extubation failure. Intensive Care Med. 2016;42(5):853-61.

7. Dres M, Dube BP, Mayaux J, Delemazure J, Reuter D, Brochard L, et al. Coexistence and impact of limb muscle and diaphragm weakness at time of liberation from mechanical ventilation in medical intensive care unit patients. Am J Respir Crit Care Med. 2017;195(1):57-66.

8. Thille AW, Cabello B, Galia F, Lyazidi A, Brochard L. Reduction of patientventilator asynchrony by reducing tidal volume during pressure-support ventilation. Intensive Care Med. 2008;34(8):1477-86.

9. Blanch L, Villagra A, Sales B, Montanya J, Lucangelo U, Lujan M, et al. Asynchronies during mechanical ventilation are associated with mortality. Intensive Care Med. 2015;41(4):633-41.

10. Younes M. Proportional assist ventilation, a new approach to ventilatory support. Theory. Am Rev Respir Dis. 1992;145(1):114-20.

11. Sinderby C, Navalesi P, Beck J, Skrobik Y, Comtois N, Friberg S, et al. Neural control of mechanical ventilation in respiratory failure. Nat Med. 1999;5(12):1433-6.

12. Sinderby C, Beck J. Proportional assist ventilation and neurally adjusted ventilatory assist - better approaches to patient ventilator synchrony? Clin Chest Med. 2008;29(2):329-42.

13. Akoumianaki E, Dousse N, Lyazidi A, Lefebvre JC, Graf S, Cordioli RL, et al. Can proportional ventilation modes facilitate exercise in critically ill patients? A physiological cross-over study: pressure support versus proportional ventilation during lower limb exercise in ventilated critically ill patients. Ann Intensive Care. 2017;7(1):64.

14. Kacmarek RM. Proportional assist ventilation and neurally adjusted ventilatory assist. Respir Care. 2011:56(2):140-8 (discussion 9-52)

15. Schmidt M, Kindler F, Cecchini J, Poitou T, Morawiec E, Persichini R, et al. Neurally adjusted ventilatory assist and proportional assist ventilation both improve patient-ventilator interaction. Crit Care. 2015;19:56.

16. Liberati A, Altman DG, Tetzlaff J, Mulrow C, Gotzsche PC, loannidis JP, et al. The PRISMA statement for reporting systematic reviews and meta-analyses of studies that evaluate health care interventions: explanation and elaboration. Ann Intern Med. 2009;151(4):W65-94.

17. Thille AW, Rodriguez P, Cabello B, Lellouche F, Brochard L. Patient-ventilator asynchrony during assisted mechanical ventilation. Intensive Care Med. 2006;32(10):1515-22.

18. Higgins J, Green S. Cochrane handbook for systematic reviews of interventions. Chichester: Wiley; 2008.

19. Wan X, Wang W, Liu J, Tong T. Estimating the sample mean and standard deviation from the sample size, median, range and/or interquartile range. BMC Med Res Methodol. 2014;14:135.

20. DerSimonian R, Laird N. Meta-analysis in clinical trials. Control Clin Trials. 1986;7(3):177-88.

21. Higgins JP, Thompson SG, Deeks JJ, Altman DG. Measuring inconsistency in meta-analyses. BMJ. 2003;327(7414):557-60.

22. Xirouchaki N, Kondili E, Vaporidi K, Xirouchakis G, Klimathianaki M, Gavriilidis $\mathrm{G}$, et al. Proportional assist ventilation with load-adjustable gain factors in critically ill patients: comparison with pressure support. Intensive Care Med. 2008;34(11):2026-34.

23. Elganady A, Beshey B, Abdelaziz A. Proportional assist ventilation versus pressure support ventilation in the weaning of patients with acute exacerbation of chronic obstructive pulmonary disease. Egypt J Chest Dis Tuberc. 2014;63(3):643-50.

24. Bosma KJ, Read BA, Bahrgard Nikoo MJ, Jones PM, Priestap FA, Lewis JF. A pilot randomized trial comparing weaning from mechanical ventilation on pressure support versus proportional assist ventilation. Crit Care Med. 2016:44(6):1098-108.

25. Demoule A, Clavel M, Rolland-Debord C, Perbet S, Terzi N, Kouatchet A, et al. Neurally adjusted ventilatory assist as an alternative to pressure support ventilation in adults: a French multicentre randomized trial. Intensive Care Med. 2016;42(11):1723-32

26. Di Mussi R, Spadaro S, Mirabella L, Volta CA, Serio G, Staffieri F, et al. Impact of prolonged assisted ventilation on diaphragmatic efficiency: NAVA versus PSV. Crit Care. 2016;20:1.

27. Kuo NY, Tu ML, Hung TY, Liu SF, Chung YH, Lin MC, et al. A randomized clinical trial of neurally adjusted ventilatory assist versus conventional 
weaning mode in patients with COPD and prolonged mechanical ventilation. Int J Chron Obstruct Pulmon Dis. 2016;11:945-51.

28. Botha J, Green C, Carney I, Haji K, Gupta S, Tiruvoipati R. Proportional assist ventilation versus pressure support ventilation in weaning ventilation: a pilot randomised controlled trial. Crit Care Resusc. 2018;20(1):33-40.

29. Colombo D, Cammarota G, Bergamaschi V, De Lucia M, Corte FD, Navalesi P. Physiologic response to varying levels of pressure support and neurally adjusted ventilatory assist in patients with acute respiratory failure. Intensive Care Med. 2008:34(11):2010-8.

30. Spahija J, de Marchie M, Albert M, Bellemare P, Delisle S, Beck J, et al. Patient-ventilator interaction during pressure support ventilation and neurally adjusted ventilatory assist. Crit Care Med. 2010;38(2):518-26.

31. Patroniti N, Bellani G, Saccavino E, Zanella A, Grasselli G, Isgro S, et al. Respiratory pattern during neurally adjusted ventilatory assist in acute respiratory failure patients. Intensive Care Med. 2012;38(2):230-9.

32. Doorduin J, Sinderby CA, Beck J, van der Hoeven JG, Heunks LM. Assisted ventilation in patients with acute respiratory distress syndrome: lungdistending pressure and patient-ventilator interaction. Anesthesiology. 2015;123(1):181-90.

33. Carteaux G, Cordoba-Izquierdo A, Lyazidi A, Heunks L, Thille AW, Brochard L. Comparison between neurally adjusted ventilatory assist and pressure support ventilation levels in terms of respiratory effort. Crit Care Med. 2016;44(3):503-11.

34. Costa R, Navalesi P, Cammarota G, Longhini F, Spinazzola G, Cipriani F, et al. Remifentanil effects on respiratory drive and timing during pressure support ventilation and neurally adjusted ventilatory assist. Respir Physiol Neurobiol. 2017:244:10-6.

35. Ferreira JC, Diniz-Silva F, Moriya HT, Alencar AM, Amato MBP, Carvalho CRR. Neurally adjusted ventilatory assist (NAVA) or pressure support ventilation (PSV) during spontaneous breathing trials in critically ill patients: a crossover trial. BMC Pulm Med. 2017;17(1):139.
36. Georgopoulos D, Prinianakis G, Kondili E. Bedside waveforms interpretation as a tool to identify patient-ventilator asynchronies. Intensive Care Med. 2006;32(1):34-47.

37. Bosma K, Ferreyra G, Ambrogio C, Pasero D, Mirabella L, Braghiroli A, et al. Patient-ventilator interaction and sleep in mechanically ventilated patients: pressure support versus proportional assist ventilation. Crit Care Med. 2007;35(4):1048-54.

38. Girard TD, Kress JP, Fuchs BD, Thomason JW, Schweickert WD, Pun BT, et al. Efficacy and safety of a paired sedation and ventilator weaning protocol for mechanically ventilated patients in intensive care (Awakening and Breathing Controlled trial): a randomised controlled trial. Lancet. 2008;371(9607):126-34

39. Strom T, Martinussen T, Toft P. A protocol of no sedation for critically ill patients receiving mechanical ventilation: a randomised trial. Lancet. 2010;375(9713):475-80

40. Carteaux G, Mancebo J, Mercat A, Dellamonica J, Richard JC, AguirreBermeo $\mathrm{H}$, et al. Bedside adjustment of proportional assist ventilation to target a predefined range of respiratory effort. Crit Care Med. 2013;41(9):2125-32.

41. Goligher EC, Dres M, Fan E, Rubenfeld GD, Scales DC, Herridge MS, et al. Mechanical ventilation-induced diaphragm atrophy strongly impacts clinical outcomes. Am J Respir Crit Care Med. 2018;197(2):204-13.

42. Zambon M, Beccaria P, Matsuno J, Gemma M, Frati E, Colombo S, et al. Mechanical ventilation and diaphragmatic atrophy in critically ill patients: an ultrasound study. Crit Care Med. 2016;44(7):1347-52.

43. Akoumianaki E, Prinianakis G, Kondili E, Malliotakis P, Georgopoulos D. Physiologic comparison of neurally adjusted ventilator assist, proportional assist and pressure support ventilation in critically ill patients. Respir Physiol Neurobiol. 2014;203:82-9.

44. Richens A. Proof of efficacy trials: cross-over versus parallel-group. Epilepsy Res. 2001;45(1-3):43-7 (discussion 9-51).

\section{Submit your manuscript to a SpringerOpen ${ }^{\odot}$ journal and benefit from:}

- Convenient online submission

- Rigorous peer review

- Open access: articles freely available online

- High visibility within the field

- Retaining the copyright to your article

Submit your next manuscript at $\boldsymbol{\nabla}$ springeropen.com 